\title{
ECO-EFICIENCIA EN LA GESTIÓN DE RESIDUOS MUNICIPALES EN CATALUNYA
}

\author{
Eco-efficiency in the urban \\ waste management at Catalunya
}

Envio 18.04.08 / Aceite 10.07.08

\section{Guillermo Javier Díaz Villavicencio ${ }^{1}$ Simone Regina Didonet ${ }^{2}$}

\section{Resumen}

El objetivo del presente trabajo es medir la eco-eficiencia en la gestión de residuos urbanos en Catalunya, España. Para ello, se presenta un modelo que incluye aspectos de medición ambiental y Análisis Envolvente de Datos DEA (Data Envelopment Análisis). La base de esta investigación, se centra en la orientación hacia la sostenibilidad, indicada por el World Business Council for Sustainable Development (WBCSD), quien también recomienda la aplicabilidad de la eco-eficiencia, dentro de los organismos públicos. A tal efecto, hemos realizado una aproximación empírica tomando una muestra de 48 municipios de Catalunya, España. Los resultados obtenidos demuestran que en términos generales, sólo existirían dos municipios eco-eficientes, lo que denotaría que existe un alto nivel de ineficiencia en materia de gestión de residuos municipales. Por otro lado, las conclusiones apuntan a demandar de parte de los órganos de gobierno, más acceso y calidad de información.

Palabras clave: Eco-eficiencia; Desarrollo Sostenible; Residuos Municipales; DEA.

\footnotetext{
${ }^{1}$ Doutorando em Estudos Empresariais, Universidad de Barcelona/Espanha; mestre em Investigação em Economia de Empresas, Universidad Autónoma de Barcelona/Espanha; graduado em Administração de Empresas, Universidad de Antofagasta, Chile. Endereço: Pasaje Las Encinas, 1630, Edificio El Sauce, 201 - Parque Inglés - Antofagasta, Chile. E-mail: guillermojavier.diaz@uab.es. Fone: 005655548195.

2Doutora em Administração, CEPEAD/UFMG; mestre em Engenharia de Produção, PPGEP/UFSM; graduada em Administração, UNIJUI; Professora Assistente na Facultad de Economía y Administración da Universidad Católica del Norte, Chile. Endereço: Facultad de Economía y Administración, Universidad Católica del Norte. Avenida Angamos, 0610, Antofagasta/Chile. E-mail: sdidonet@ucn.cl. Telefone: 005655355 755. Fax: 005655355801.
} 


\begin{abstract}
The purpose of this study is to measure the eco-efficiency in the urban waste management at Catalunya, Spain. For it, we present a model that includes environmental aspects of measurement and data envelopment analysis (DEA). The basis of this research focuses on the direction towards sustainability, as indicated by the World Business Council for Sustainable Development (WBCSD), who also recommends the applicability of eco-efficiency within government agencies. For this, we have made an empirical approximation taking a sample of 48 municipalities in Catalunya, Spain. The results show that in general terms there are only two municipalities eco-efficient, which means that there is a high level of inefficiency in the urban waste management. On the other hand, the findings pointing that the government has to improve the access and the quality of the information.
\end{abstract}

Keywords: ECo-Efficiency; Sustainable Development; Urban Waste; DEA.

\title{
Introducción
}

En los temas medioambientales, siempre salta la duda si estamos haciendo las cosas bien o mal, desde un punto de vista de la acción o de concienciación social, en pro de un desarrollo sostenible. En tal sentido, la búsqueda de respuestas a tales interrogantes, apuntan directamente a los agentes que tienen en mayor medida la producción de bienes y servicios. En este caso, no se escapa ninguna organización, sea ésta con o sin fines de lucro. Frente a esta perspectiva Porter y Van Der Linde (1995) demuestran que, desde un punto de vista restrictivo, la aplicación de estándares medioambientales, encuentran respuestas positivas y altamente competitivas en las empresas, empujando a las firmas a utilizar recursos más productivos, siendo más innovadoras y aplicando una lógica ambiental.

Frente a la medición de estándares medioambientales, están las regulaciones que nos pueden clarificar si nuestro actuar, está dentro de lo correcto o no. En tal sentido, las regulaciones juegan un papel muy importante. Hernández, Picazo y Reig (1997), señalan que son muchas y ciertamente dispersas, las aportaciones que tratan de analizar el impacto de estas regulaciones ambientales, sobre el comportamiento tanto de empresas privadas como de organismos públicos.

Desde una perspectiva de generación de residuos, podemos decir que en la Unión Europea se generan entre 3,5 y 7 toneladas aproximadamente de residuos por habitante al año, incluyendo los residuos industriales, de la construcción y municipales. El informe AEMA (2004), señala que, sólo en el tema de generación de residuos de embalajes, entre los periodos 1997 y 2001 hubo un aumento de un 7\% del total. Considerando sólo los datos que de manera obligatoria exige la UE a los estados miembros que son; cantidad de plástico, cristales, metal y papel. Vemos que frente a esta realidad, sin considerar otros elementos como pilas, aceites de todo tipo, gases, etc. nos enfrentamos a que, el tema en cuestión reviste una preocupación mayor y sitúa a la problemática en un contexto global.

En España, es evidente que la inserción en la Comunidad Económica Europea, obliga a la generación de nuevas y más restrictivas normas, con relación a la temática medioambiental. Por ello, España desde el punto de vista legal, generó la Ley 10/1998, del 21 de abril, de residuos (BOE n. 96, de 22-04-1998) ${ }^{1}$ que en su articulo 1 señala: "esta ley tiene por objeto prevenir la

${ }^{1}$ Los BOE y las leyes relacionadas, se encuentran en la web del Ministerio de Justicia de España o en webs relacionadas. 
producción de residuos, establecer el régimen jurídico de su producción y gestión y fomentar, por este orden, su reducción, su reutilización, reciclado y otras formas de valorización, así como regular los suelos contaminados, con la finalidad de proteger el medio ambiente y la salud de las personas".

Desde el punto de vista de residuos domiciliarios en particular, en la comunidad autónoma de Cataluña, ha aumentado en un 38\% la generación de residuos municipales, en los últimos 6 años (1998-2004) según, los estudios realizados por el centro Catalán del Reciclaje de la Agencia de Residuos de Catalunya. Por otro lado, el programa de gestión de residuos municipales 2001-2006 (PROGREMIC), no establece objetivos cuantitativos propios de prevención, ${ }^{2}$ delatando la falta de medidas y/o herramientas de medición, que se deben tener para la prevención del tema en cuestión.

Una de las herramientas que nos pueden ayudar a medir la problemática medioambiental, con resultados finales objetivos, es la medición de la eficiencia desde un punto de vista medioambiental, a saber la "eco-eficiencia".

La "eco-eficiencia", es un término que nace como respuesta a la necesidad de indicadores de productividad y eficiencia, en los procesos productivos limpios o verdes, y que para el presente estudio, nos servirá para medir la eficiencia de un área de alto impacto ambiental, como es, el de los desechos o residuos generados por la sociedad, y administrados por los gobiernos locales o ayuntamientos, satisfaciendo así, la necesidad de información objetiva para la toma de decisiones. Para ello, estudiaremos a quienes tienen la responsabilidad de gestionar los impactos ecológicos, en determinados territorios catalanes, a saber, las Administraciones Municipales, que son organismos públicos, sin fines de lucro y con un alto impacto ciudadano, desde la perspectiva ambiental.

En el área de la recogida de desechos domiciliarios, los municipios en su toma de decisiones, influyen en los impactos ecológicos, sanitarios, culturales, turísticos y otros.

La finalidad de este estudio, es que sea extrapolable (generalizable a otras realidades), para tener un patrón de referencia, y ver si la eco-eficiencia de los municipios catalanes, es un referente o pauta comparativa a seguir, por otras regiones del estado Español, o de otras regiones que tengan políticas de gestión de residuos municipales.

\section{Marco conceptual}

La administración municipal, es una unidad productiva de servicios sociales. En tal sentido, la llamada teoría neoclásica de la empresa, considera a la empresa (organización), como la unidad de producción elemental, maximizadora de beneficio, que ejerce de demandante de factores productivos y, a su vez, de oferente de bienes y servicios (SALAS, 1984). Así, " la empresa se encargaría de transformar factores (inputs) en productos (outputs), según una determinada función de producción. La cual define, el máximo producto alcanzable con una cierta cantidad de factores - aquella que, minimiza los costes de producción a los precios corrientes de dichos factores - y que, viene determinada por el estado del conocimiento técnico" (RIALP, 2003, p.54). Por ello, esta teoría podría aplicarse tanto a empresas y/o organismos públicos, con o sin fines de lucro, en busca de la eficiencia.

En términos de la relación inputs x outputs se habla de la eficiencia. Álvarez (2001) señala que, existen tres tipos de eficiencia; a) eficiencia de escala: cuando una empresa está produciendo en una escala de tamaño óptimo, que es la que permite maximizar el beneficio;

${ }^{2}$ Pag. 32 Acciones de prevención de residuos municipales promulgados para las localidades de Catalunya y Europa.

Rev. Adm. UFSM, Santa Maria, v. 1, n. 2, p. 193-208, mai./ago. 2008 
b) eficiencia asignativa: cuando la empresa combina los inputs en la proporción que minimiza su coste de producción; c) eficiencia técnica: cuando la empresa obtiene el máximo output posible, con la combinación de inputs empleados.

Tales conceptos también pueden ser considerados bajo la perspectiva medioambiental. En ese caso la discusión enfocaría en la economía medioambiental, la cual según FIELD (2001), se sitúa en la línea de la microeconomía, se concentra principalmente en cómo y por qué, las personas toman decisiones que tienen consecuencias ambientales. Además, se ocupa de estudiar las maneras de cómo, se pueden cambiar las políticas e instituciones económicas, con el propósito de equilibrar un poco más esos impactos ambientales, con los deseos humanos y las necesidades del ecosistema en si mismo. En tal sentido, podemos tomar este marco conceptual, como referencia de nuestro trabajo, ya que, combina políticas ambientales con las necesidades del entorno natural y humano.

De ahí nace el concepto de eco-eficiencia, propuesto originalmente por el Concejo Mundial de Negocios para el Desarrollo Sostenible en 1992, World Business Council for Sustentainable Development (WBCSD). Éste, como un concepto de negocios aplicable a las empresas privadas, sin embargo, podemos aplicar el concepto a las administraciones públicas (gerencia pública), por que son unidades productivas de servicios sin fines de lucro, tal como lo recomienda el WBCSD. En si el concepto señala que la eco-eficiencia se obtiene por medio del suministro de bienes y servicios a precios competitivos, que satisfagan las necesidades humanas y proporcionen calidad de vida, mientras progresivamente reducen los impactos ecológicos y el consumo de recursos a lo largo de su ciclo de vida.

En la Figura 1, Erkko, Melanen y Mickwitz (2005) nos confieren una formulación sobre el concepto de eco-eficiencia, de esta manera, queda entendido que el valor agregado económico que generan o buscan las empresas, es sin duda, el factor que debe maximizarse frente a un denominador de impacto ecológico agregado, el cual debe disminuirse.

A tal razón, a mayor valor económico agregado y menor impacto medioambiental, el índice de eco-eficiencia será más alto.

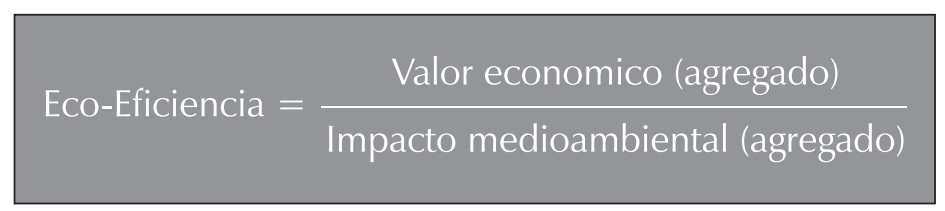

Fuente: Erkko, Melanen y Mickwitz (2005, p. 799)

Figura 1 - Fórmula de eco-eficiencia.

Bajo esta perspectiva, la empresa que desee maximizar su eco-eficiencia, podrá hacerlo aumentando el numerador (según Figura 1), en mayor proporción que el denominador o disminuyendo este último. Pero, se podría estimar que es más conveniente plantear la minimización de la relación inversa, ya que bajo el contexto del Desarrollo Sostenible, el objetivo debería orientarse más, a minimizar los impactos que, ha maximizar el valor de la producción.

El hecho de poder evaluar técnicamente a los municipios, a través de la metodología de Análisis Envolvente de Datos DEA (Data Envelopment Analysis), propuesto originalmente por Charnes, Cooper y Rhodes (1978), sitúa en sí, a los municipios dentro de los mismos parámetros técnicos de medición que una empresa privada. En tal sentido, las administraciones municipales, pasarían a ser nuestras Unidades de Toma Decisión o DMUs (Decision Making Units). En línea con lo que plantea Farell (1957), se podrá generar una frontera no paramétrica de eficiencia, bajo un rendimiento constante de escala. 
La teoría del DEA, es aplicable con una orientación a maximizar los output considerando output deseados y output indeseados, manteniendo o minimizando el último output. Como referencia tenemos los trabajos de Zofío y Prieto (2001), Bevilacqua y Braglia (2002), Côte, Booth y Louis (2005) y Jollands, Lermit y Patterson (2004) desde la perspectiva de cómo afectan distintas industrial a los países o grandes áreas. Courcelle, Kestemont y Tyteca (1998), Eriksson et al. (2005) y Rovira y Prior (2004) desde la óptica de la aplicación del DEA a los residuos de los municipios o comunidades. Desde una perspectiva de tratamiento de output indeseados, en las empresas privadas o grandes sectores productivos, destacan los trabajos metodológicos de Chung, Färe y Grosskopf (1997), utilizando función de distancia direccional, robustamente estudiada hoy en día bajo la perspectiva de las regulaciones ambientales, como es el caso de Picazo, Reig y Hernández (2005). También los trabajos de Korhonen y Luptacik (2004) en línea con la eco-eficiencia y finalmente Tyteca (1997) con indicadores de performance medioambiental por citar algunos.

Para tener un criterio estándar, del proceso productivo de los inputs/outputs que ingresan al sistema de medición de la eco-eficiencia, debemos considerar que existen tres tipos de rendimientos; los buenos (positivos), malos (negativos) y neutrales. Donde el valor positivo, necesariamente no significa una ventaja monetaria, sino una salida positiva, como el reciclage de materiales, o un producto deseado como energía eléctrica o calor. Por otro lado el valor negativo significa, una salida dañina al medio ambiente, como las aguas contaminadas. Los valores neutrales, en este caso, serian aquellos que no producen daño significativo al medioambiente, como el calor que desecha una planta eléctrica, según el caso que ilustra Allen (1999). ${ }^{3}$

En tal sentido, se dan seis tipo de categorías de conveniencias ecológicas diferentes, según Dyckhoff y Allen (2001) estas serian: factor, por factores, reductores, producto, por productos y contaminantes. Ver Cuadro 1.

\begin{tabular}{|l|l|l|l|}
\hline \multicolumn{1}{|r|}{$\begin{array}{r}\text { Clasificacion de } \\
\text { objetivo }\end{array}$} & \multicolumn{1}{|c|}{ Bueno } & \multicolumn{1}{c|}{ Neutral } & \multicolumn{1}{c|}{ Malo } \\
\cline { 1 - 3 } Input & Factor & Por-factores & Reductores \\
\hline Output & Producto & Por-productos & Contaminantes \\
\hline
\end{tabular}

\begin{tabular}{|l|l|l|}
\hline & & \\
\hline Deseable & Indiferente & Indeseable \\
\hline
\end{tabular}

Fuente: Allen (1999).

Cuadro 1 - Categorización de objetivos ecológicamente pertinentes según "caso normal"

Seguidamente, se deben considerar los indicadores de medición medioambiental environmental performance indicators (EPIs.), señalados por Courcelle, Kestemont y Tyteca (1998), Allen (1999), Burritt y Saka (2005) entre otros. Estos indicadores, pueden ser expresadas en términos absolutos o en términos relativos, con el objetivo de medir cuantitativamente los aspectos medioambientales, basados en unidades monetarias o no monetarias (el coste de

${ }^{3}$ Allen (1999, p. 211) nos detalla en un ejemplo de aplicación de DEA tradicional de una planta de generación eléctrica. Fig.3.2 Categorisation for a waste heating power plant. 
residuos, la cantidad expresada de residuos en toneladas, el coste de residuos para reciclar/ cantidad de residuos). Los EPIs, son integrados en varios instrumentos de control ecológico. Pero ellos, también representa un instrumento de información y de planificación muy útil, para hacer comparaciones en el tiempo o proyectos de Benchmarking.

Por otro lado, debemos tener claridad sobre los factores que influirán en el modelo que se usará para una medición, ya que, la literatura nos entrega a lo menos cuatro grandes factores o indicadores de sustentabilidad, para la medición de gestión de residuos.

Según Courcelle, Kestemont y Tyteca (1998), los factores serian divididos a corto y largo plazo, viendo los aspectos económicos, medioambientales, sociales, culturales y técnicos. Ver Cuadro 2.

Cuadro 2 - Ejemplos de factores requeridos en la estructuración de indicadores para la gestión de residuos

\begin{tabular}{|c|c|c|}
\hline Aspectos & $\begin{array}{l}\text { Indicador a Corto } \\
\text { plazo }\end{array}$ & Indicador a Largo plazo \\
\hline Económicos & $\begin{array}{l}\text { Costos de inversión } \\
\text { Costo neto de } \\
\text { operación } \\
\text { Costo neto total por la } \\
\text { tonelada reunida } \\
\text { Costo total anual neto } \\
\text { Mercado potencial }\end{array}$ & $\begin{array}{l}\text { Viabilidad a largo plazo de búsqueda y } \\
\text { ordenación de operaciones futuras. }\end{array}$ \\
\hline Medioambientales & $\begin{array}{l}\text { Cantidad } \\
\text { Calidad de } \\
\text { reconversión de } \\
\text { material } \\
\text { El residuo } \\
\text { La polución } \\
\text { El ruido } \\
\text { El uso de basural } \\
\text { El uso de recursos } \\
\text { naturales }\end{array}$ & $\begin{array}{l}\text { Impacto global: perdida de biodiversidad, } \\
\text { peligros globales, deposiciones ácidas, } \\
\text { paisajismo. }\end{array}$ \\
\hline $\begin{array}{l}\text { Sociales y } \\
\text { culturales }\end{array}$ & $\begin{array}{l}\text { Aceptación pública } \\
\text { Participación } \\
\text { Nivel de salud pública } \\
\text { Empleos }\end{array}$ & $\begin{array}{l}\text { Bienestar, la disponibilidad de recursos } \\
\text { naturales (materia \& energía). }\end{array}$ \\
\hline Técnicos & $\begin{array}{l}\text { Escala } \\
\text { Flexibilidad }\end{array}$ & Futuro desarrollo potencial. \\
\hline
\end{tabular}

Fuente: Adaptado de Courcelle, Kestemont y Tyteca (1998). 
Debemos considerar que existen cinco maneras de tratar el output indeseado, según Seiford y Zhu (2002), señalan que la primera de ellas es ignorar los output indeseados, así serán considerados solamente outputs deseados. La segunda opción, es insertar el output indeseado dentro de los cálculos, considerando al output indeseado como output normal. En la tercera opción, está el considerar el output indeseado como input. Los mismos autores, señalan que este procedimiento no refleja el verdadero proceso de producción. Según Färe et al. (1989) esta sería una manera de eliminar el efecto negativo del output. En el caso de la cuarta opción, los inputs se miden sólo con el output indeseado, esto significa que en los análisis los resultados deben ser interpretados de manera inversa, ya que la DMU que obtenga un score $=1,0$ será la más eficiente, en la generación de output no deseados. Finalmente, en el quinto caso, se debe transformar los valores del output indeseado, elevados a la potencia ${ }^{(-1)}$ pasando de valor negativo a positivo, dentro del proceso productivo. En tal sentido, se utilizan las variables adaptadas como output, interpretando los resultados (scores) de manera normal.

\section{Modelo}

Para evaluar la eco-eficiencia de los residíos municipales, proponemos un modelo que puede ir adaptándose, en la medida que se obtengan datos relevantes, dentro de la investigación que se pueda realizar. En tal sentido, el modelo considera como elemento esencial, el seguir etapas predeterminadas. Para ello, se propone un modelo dinámico, que abarca las exigencias mínimas expresadas por Allen (1999), separando inputs y outputs deseados e indeseados, dentro de un aspecto medioambiental básico, como señala Courcelle, Kestemont y Tyteca (1998).

Este modelo, nos orientará hacia la formulación de un indicador de eco-eficiencia medioambiental, orientado al output, seleccionando los indicadores medioambientales (EPIs) señalados por Tyteca (1996).

El modelo propuesto considera tres etapas, en la primera etapa se debe:

a) Determinar el número de DMUs, y realizar una correlación de las variables, considerando las correlaciones no paramétricas, índice Tau_b de Kendall;

b) Selección de input y output en deseables, indeseables e independientes. Según los aspectos a medir;

c) Considerar el EPI (cantidad de residuos seleccionados/cantidad total de residuos) como performan que adopta el modelo.

En una segunda etapa, se aplican los modelos DEA orientados al output, poniendo a prueba las orientaciones entregadas por Seiford y Zhu (2002), considerando para este trabajo tres modelos (M_DEA). M_1 input y output deseados. M_2 inputs sólo, con el output indeseados y M_3 introducir output indeseado como ${ }^{(-1)}$. Todo dentro de un modelo general llamado "Ecoeficiencia para la gestión de residuos municipales" (Figura 2). 


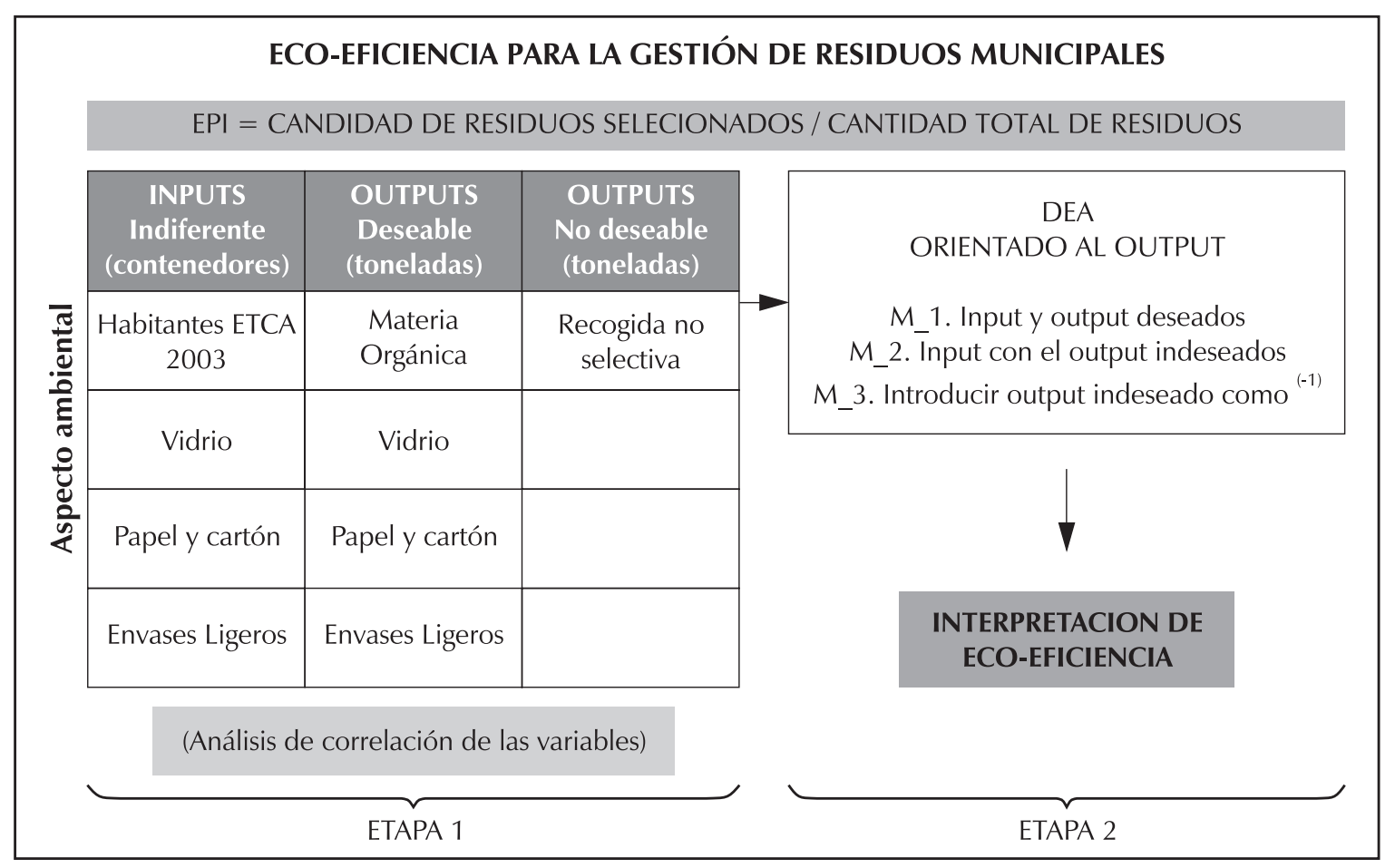

Fuente: eleboración propia a partir de ALLEN (1999), COURCELLE, KESTEMONT y TYTECA (1998) y TYTECA (1996)

Figura 2 - Modelo de Eco-Eficiencia para la Gestión de Residuos Municipales.

En la tercera etapa, interpretación los resultados de la eco-eficiencia, se podrán generar rankings, entre los Scores de eficiencia, señalando cuales son los municipios ineficientes y con cuales deben compararse para ser eficientes (benchmark).

\section{Metodología}

Según los datos que nos proporcionará el DEA, por la aplicación del Modelo de ECo-Eficiencia para la Gestión de Residuos Municipales, podremos ver: la eficiencia de cada municipio frente a la gestión de residuos municipales; cuál es el municipio de referencia al cual debe seguir; y cuánto debe mejorar (según los resultados), cada municipio para poder alcanzar la eco-eficiencia.

La primera propuesta M_1, es ignorar los output indeseados. Así serán considerados solamente outputs deseados, generando un análisis clásico de DEA, el cual nos dirá dentro de una frontera no paramétrica, cuales son los municipios eficientes. Luego, se tiene:

\section{M_1 = INPUTS + OUTPUTS DESEADOS}

Para el caso del M_2, el input (Habitantes), se incorpora sólo con el output indeseado, por que los otros inputs no generan output indeseados. Por tanto, si los incluimos en el cálculo generarán resultados erróneos. En tal sentido, esto significa que, en nuestro análisis los resultados deben ser interpretados de manera inversa, ya que, el municipio que obtenga el score 1,0, será el más eficiente en la generación de output no deseados, por lo tanto será el más ineficiente en la recogida selectiva. Luego, 


\section{M_2 = INPUT (Habitantes) + OUTPUT INDESEADO}

En el M_3, se debe transformar los valores del output indeseado, elevándolos a potencia ${ }^{(-1)}$ pasando de valor negativo a positivo, dentro del proceso productivo. En tal sentido, utilizaremos las variables adaptadas como output normal, interpretando los resultados (scores) de manera normal. Así tenemos:

$$
\text { M_3 = INPUTS + OUTPUTS DESEADOS + OUTPUT INDESEADO }{ }^{(-1)}
$$

Para el caso particular de este trabajo, los modelos DEA parten de la base de la eficiencia técnica de cada DMU, para cada modelo M_1, M_2 y M_3, es dada por la resolución del siguiente problema de programación lineal, adaptado de Färe, Grosskopf y Lovell (1994):
M_1
M_2
M_3

$\operatorname{Max} \theta$

$\operatorname{Max} \theta$

S.t.

$\sum_{j=1}^{N} \lambda_{j} y_{s j} \geqq \theta y_{s i} ; s=1, \ldots s$

$\sum_{j=1}^{N} \lambda_{j} x_{m j} \leqq x_{m i} ; m=1, \ldots M$

$\lambda_{j} \geqq 0 ; j=1, \ldots N$
S.t.

$\lambda_{\mathrm{j}=1}^{\mathrm{N}} \lambda_{\mathrm{j}} \mathrm{y}_{\mathrm{sj}} \geqq \theta \mathrm{y}_{\mathrm{si}}^{*} ; \mathrm{s}=1, \ldots \mathrm{s}$

$\sum_{j=1}^{N} \lambda_{j} x_{m j} \leqq x_{m i} ; m=1, \ldots M$

$\lambda_{j} \geqq 0 ; j=1, \ldots N$
$\operatorname{Max} \theta$

S.t.

$$
\begin{aligned}
& \lambda_{j=1}^{N} \lambda_{j} y_{s j} \geqq \theta y_{s i} ; s=1, \ldots s \\
& { }_{j=1}^{N} \lambda_{j} y^{*}{ }_{m j}^{-1} \leqq \theta y^{*}{ }_{s i}^{-1} ; s=1, \ldots S \\
& \sum_{j=1}^{N} \lambda_{j} x_{m j} \leqq x_{m i} ; m=1, \ldots M \\
& \lambda_{j} \geqq 0 ; j=1, \ldots N
\end{aligned}
$$

Donde el municipio $j$ emplea un vector de input $x=\left(x_{1}, \ldots x_{1}, \ldots x_{n}\right) \in R^{n}{ }_{+}$a fin de producir un output $y=\left(y_{1}, \ldots y_{j_{1}} \ldots y_{n}\right) \in R^{n}{ }_{+}$. Así, una DMU será eficiente, en la perspectiva de Farell (1957) si $\theta^{*}=1$ y ineficiente si $0<\theta^{*}<1$, considerando que los retornos de escala sean constantes (BANKER et al., 1989) para el caso de M_3 $y^{*}=$ output indeseado.

En el caso del tratamiento tradicional de eficiencia (Figura 3), tenemos gráficamente, el máximo output (ga) que puede obtener una DMU, cuando los output indeseados no son considerados, es ga dentro de la frontera seria el punto $A$. 


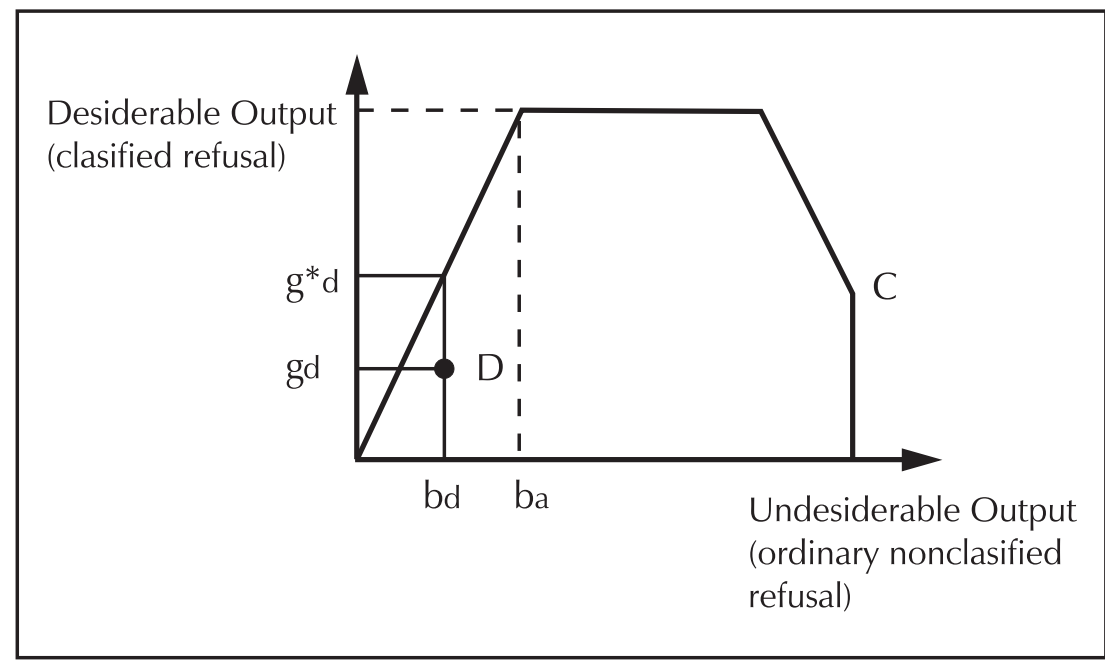

Fuente: Adaptado de Tyteca (1997), Rovira y Prior (2004)

Figura 3 - Modelo de orientación al output con output indeseado.

Suponiendo que no exista ninguna modificación, en los atributos del output indeseado, el máximo output que se puede obtener es $g^{*} d$ En consecuencia, parte del aumento potencial en nivel de output (ga- $g^{*} d$ ), es imposible de ser alcanzado sin aceptar algún deterioro en los padrones de calidad (aumentar los output indeseados). En otras palabras, el control de output indeseado, requiere el consumo de recursos que podrían ser empleados en la producción de output deseados.

\section{Muestra del estudio}

En Catalunya residen 6.813.319 habitantes (INE 2005), repartidos en 946 municipios, siendo Sant Jaume de Frontanyà con 27 habitantes el menor, y Barcelona con 1.578.546 habitantes el mayor municipio.

Hemos seleccionado 48 municipios (DMUs), que cumplen con la condición de tener más de 20 mil habitantes y que representan un total de 4.463 .751 habitantes, constituyendo un $66 \%$ de la población de Cataluña, según ETCA 2003. Se considera el índice ETCA por que asume el total de la población, incluyendo la población equivalente, más los habitantes por pernoctación en hoteles. Este índice se publica desde 1998 en periodos de 5 años.

Para el cálculo de la frontera eco-eficiente, serán considerados rendimientos constantes de escala, según el modelo CCR (CHARNES, COOPER y RHODES, 1978) con orientación al output, dado que, lo que se desea, es aumentar al máximo las cantidades de outputs deseados, mientras se controla las cantidades de outputs indeseados. En tal sentido, se ha utilizado como herramienta de cálculo, el programa EMS, validando los resultados en Solver de Excel.

En cuanto a las variables (inputs y outputs) utilizadas tenemos:

- Inputs: de recogida selectiva de residuos.

- Habitantes ETCA 2003;

- Vidrio (contenedores);

- Papeles y cartones (contenedores);

- Envases ligeros (contenedores). 
Outputs deseados: residuos de recogida selectiva (toneladas).

- Materia orgánica;

- Vidrio;

- Papel y cartón;

- Envases ligeros.

Outputs indeseados: residuos de recogida no selectiva (toneladas).

- Total recogida restante de residuos, directa a disposición final.

\section{Resultados}

La verificación de las correlaciones entre las variables, utilizando el SPSS, nos indica que no hay variables relacionadas con valor igual a 1. Por lo tanto los inputs y outputs utilizados en la muestra, son posibles de analizar. Por otro lado, se ha realizado un estadístico descriptivo (Tabla 1) con el objeto de verificar que no hay datos perdidos (requisito ineludible del DEA).

La Tabla 1, nos muestra los resultados de los 3 modelos utilizados (ordenados según cantidad de habitantes de forma descendente).

Tabla 1 - Estadístico descriptivo

\begin{tabular}{|c|c|c|c|c|c|c|c|c|c|c|}
\hline & & Habitantes & Vidrio & PC & Envases & $\begin{array}{c}\text { Materia } \\
\text { Organica }\end{array}$ & Vidrio & $\begin{array}{l}\text { Papel y } \\
\text { Carton }\end{array}$ & $\begin{array}{r}\text { Envases } \\
\text { Ligeros }\end{array}$ & $\begin{array}{c}\text { Recogida No } \\
\text { Deseada }\end{array}$ \\
\hline \multirow[b]{2}{*}{$\mathbf{N}$} & Válidos & 48 & 48 & 48 & 48 & 48 & 48 & 48 & 48 & 48 \\
\hline & Perdidos & 0 & 0 & 0 & 0 & 0 & 0 & 0 & 0 & 0 \\
\hline \multicolumn{2}{|c|}{ Desv. típ. } & 228506,29 & 359,82820 & 361,74810 & 384,93792 & 8557,654 & 2851,028 & 4599,894 & 1528,1778 & 97860,88409 \\
\hline \multicolumn{2}{|c|}{ Mínimo } & 21072,0 & 38,00 & 35,00 & 28,00 & 10,48 & 202,47 & 285,95 & 8,84 & 8038,12 \\
\hline \multicolumn{2}{|c|}{$\overline{\text { Máximo }}$} & 1608095,0 & 2508,00 & 2500,00 & 2500,00 & 59356,55 & 20153,47 & 32124,45 & 10644,02 & 690308,00 \\
\hline
\end{tabular}

Fuente: elaboración propia eficientes.

En el modelo M_1 y $M_{-}$3, los valores con un 1,00 representan los municipios

En el M_2 el criterio es inverso, por tanto los municipios eficientes son los que tienen valores sobre el percentil 1,73 según la Tabla 2, generada a partir de los propios datos de la muestra.

El criterio de eficiencia en los tres modelos nos aproximaría a una relación de Ecoeficiencia. 
Tabla 2 - Resultados de eco-eficiencia

\begin{tabular}{|c|c|c|c|c|}
\hline DMU & Municipios & M_1 & M_2 & M_3 \\
\hline $\mathrm{F} 1$ & Barcelona & 1,00 & 1,50 & 1,00 \\
\hline F2 & L'Hospitalet de Llobregat & 1,19 & 1,95 & 1,19 \\
\hline F3 & Badalona & 1,87 & 1,67 & 1,87 \\
\hline $\mathrm{F} 4$ & Sabadell & 1,20 & 1,40 & 1,20 \\
\hline $\mathrm{F5}$ & Terrassa & 1,12 & 1,61 & 1,12 \\
\hline F6 & Tarragona & 1,26 & 1,45 & 1,26 \\
\hline F7 & Lleida & 1,21 & 1,44 & 1,21 \\
\hline F8 & Mataró & 1,14 & 1,33 & 1,14 \\
\hline F9 & Santa Coloma de Gramenet & 1,85 & 1,47 & 1,85 \\
\hline F10 & Reus & 1,00 & 1,76 & 1,00 \\
\hline F11 & Girona & 1,00 & 1,50 & 1,00 \\
\hline F12 & Cornellà de Llobregat & 1,24 & 1,50 & 1,24 \\
\hline F13 & Sant Boi de Llobregat & 1,19 & 1,52 & 1,18 \\
\hline F14 & Manresa & 1,21 & 1,71 & 1,21 \\
\hline F15 & Rubí & 1,64 & 1,73 & 1,00 \\
\hline F16 & El Prat de Llobregat & 1,16 & 2,06 & 1,16 \\
\hline F17 & Vilanova i la Geltrú & 1,00 & 1,48 & 1,00 \\
\hline F18 & Granollers & 1,42 & 1,83 & 1,40 \\
\hline F19 & Viladecans & 1,62 & 1,48 & 1,58 \\
\hline F20 & Cerdanyola del Vallès & 1,26 & 1,42 & 1,26 \\
\hline F21 & lloret de mar & 1,00 & 1,23 & 1,00 \\
\hline F22 & Castelldefels & 1,16 & 1,34 & 1,16 \\
\hline F23 & Mollet del Vallès & 1,45 & 1,79 & 1,44 \\
\hline F24 & Blanes & 1,73 & 1,23 & 1,65 \\
\hline F25 & Esplugues de Llobregat & 1,11 & 1,67 & 1,09 \\
\hline F26 & Gavà & 1,43 & 1,38 & 1,39 \\
\hline F27 & Vic & 1,00 & 2,12 & 1,00 \\
\hline F28 & Sant Feliu de Llobregat & 1,27 & 1,79 & 1,23 \\
\hline F29 & Igualada & 1,19 & 1,65 & 1,19 \\
\hline F30 & Cambrils & 1,00 & 1,16 & 1,00 \\
\hline F31 & El Vendrell & 1,45 & 1,13 & 1,21 \\
\hline F32 & Vilafranca del Penedès & 1,16 & 1,58 & 1,13 \\
\hline F33 & Tortosa & 1,66 & 1,60 & 1,35 \\
\hline F34 & Sant Adrià de Besòs & 1,56 & 1,61 & 1,42 \\
\hline F35 & Ripollet & 1,18 & 1,81 & 1,18 \\
\hline F36 & Martorell & 1,53 & 2,03 & 1,34 \\
\hline F37 & Sant Joan Despí & 1,32 & 1,71 & 1,21 \\
\hline F38 & Barberà del Vallès & 1,44 & 1,79 & 1,31 \\
\hline F39 & Sitges & 1,00 & 1,00 & 1,00 \\
\hline $\mathrm{F} 40$ & Pineda de Mar & 1,00 & 1,05 & 1,00 \\
\hline F41 & Calella & 1,00 & 1,46 & 1,00 \\
\hline $\mathrm{F} 42$ & Sant Vicenç dels Horts & 1,34 & 1,33 & 1,24 \\
\hline $\mathrm{F} 43$ & Sant Pere de Ribes & 1,15 & 1,45 & 1,00 \\
\hline F44 & Sant Feliu de Guíxols & 1,20 & 1,02 & 1,00 \\
\hline $\mathrm{F} 45$ & Santa Perpètua de Mogoda & 1,27 & 1,69 & 1,15 \\
\hline F46 & Salt & 1,06 & 1,76 & 1,00 \\
\hline $\mathrm{F} 47$ & Valls & 1,04 & 1,50 & 1,00 \\
\hline F48 & Vila-seca & 1,00 & 1,35 & 1,00 \\
\hline
\end{tabular}

Fuente: elaboración propia 
Además, se ha confirmado que los resultados obtenidos son uniformes, en términos de medias y concentración de datos, como se puede observar en la Tabla 3.

Tabla 3 - Estadístico descriptivo de cada DEA aplicado

\begin{tabular}{l|l|c|c|c}
\hline \multicolumn{2}{l|}{} & DEA_1 & DEA_2 & DEA_3 \\
\hline \multirow{2}{*}{ N } & Válidos & 48 & 48 & 48 \\
\cline { 2 - 5 } & Perdidos & 0 & 0 & 0 \\
\hline \multirow{2}{*}{ Media } & 1,2558 & 1,5425 & 1,2308 \\
\hline Desv. típ. &, 23838 &, 26156 &, 23964 \\
\hline Mínimo & 1,00 & 1,00 & 1,00 \\
\hline Máximo & & 1,87 & 2,12 & 1,87 \\
\hline Percentiles & $\mathbf{2 5}$ & 1,0450 & 1,3850 & 1,0000 \\
\cline { 2 - 5 } & $\mathbf{5 0}$ & 1,1950 & 1,5000 & 1,1900 \\
\cline { 2 - 5 } & $\mathbf{7 5}$ & 1,4275 & 1,7250 & 1,4000 \\
\hline
\end{tabular}

Fuente: elaboración propia

El análisis de los resultados, según el modelo propuesto, para el M_1 nos arroja que 11 municipios alcanzan la eco-eficiencia. Cumpliendo con la formación estándar de Charnes, Cooper y Rhodes (1978), ignorando la existencia de output indeseado, la media es de 1,25. Esto significa, que los municipios podrían tener un desempeño eficiente si aumentaran en un $25 \%$ las cantidades de outputs deseados (recogida selectiva de residuos). Desde una perspectiva medioambiental, lo ideal seria que del total de output generados por el municipio, se vaciara o trasladaran, los output indeseados en deseados.

Para M_2, la interpretación se debe realizar de manera inversa. Es decir, los valores sombreados, representan a los municipios más eficientes en la generación de output indeseados. En consecuencia, se debe interpretar como los municipios menos eficientes de la muestra.

En este caso, hay un municipio con un score 1,0. Por tanto el municipio de Sitges, sería el municipio más ineficiente en nuestro análisis, bajo la lógica inversa de CCR.

Si consideramos como eficientes a los municipios, que están sobre el percentil 75 (Tabla 5), tendríamos a 12 municipios. Para nuestro análisis serian los más eficientes, en el sentido que son los que menos residuos indeseados generan.

El caso del M_3, generó 16 municipios eficientes, se interpreta este resultado, señalando que los valores del output no deseado, deben asumir una transformación decreciente (inversa), donde el valor final del output no deseado, pasa a ser positivo. Así, aplicando un vector de transformación (elevando el valor a la potencia -1), se expanden los outputs deseados y se contrae el output indeseado según Seiford y Zhu (2002) y Färe et al. (1989). 


\section{Conclusiones y limitaciones}

Según los resultados del modelo propuesto en la Figura 2, podríamos señalar que, estarían dentro de la categoría de municipios eco-eficientes los ayuntamientos de Reus y Vic. Esto es así, por que son los que simultáneamente resultan eco-eficientes, en los tres modelos aplicados. Por tanto, serian los municipios que mejor clasifican sus residuos, los que menos generan desechos no deseados, y los que en un análisis global, tienen un redimiendo óptimo, dentro de un análisis no paramétrico.

Se debe señalar que Sitges, es eficiente en selección de residuos, pero a la vez su población es la que genera más cantidad de residuos no deseados. Por otro lado es eficiente en el M_3, lo que supone, que en términos generales es un municipio eficiente, pero para este modelo propuesto no seria eco-eficiente.

Cabria preguntarse en un estudio posterior, si los resultados que en este estudio presentan los municipios catalanes, se deben a, si los factores son endógenos de orden institucional: mala supervisón de los agentes municipales, bajos presupuestos que afectan al rendimiento general del sistema, problemas de control interno de los empleados de la gestión de residuos. O son factores exógenos: demasiada presión social (ONGs, grupos de presión medioambiental), mala percepción de la comunidad hacia el municipio, excesivo control del Estado sobre el tema en cuestión, educación, factores ligados al turismo, etc.

Quizás, la gestión de los residuos urbanos se está haciendo bien, y no se podría hacer mejor, por la falta de recursos en tecnología, concienciación ciudadana, supervisión, etc.

Una limitación del estudio realizado, es que en las variables utilizadas no fue posible incluir valores económicos. El introducir variables de costes al modelo, representaría una mejora para llegar a medir la eco-eficiencia. Toda vez, que es patente el esfuerzo que hace la comunidad Catalana, en términos de inversión en gestión de residuos municipales. Siendo Cataluña una de las comunidades autónomas que más invierte en materia medioambiental.

Por último, vemos que en una futura aproximación, deberíamos incorporar mejoras metodológicas, como lo es la función de distancia direccional DDF (Direccional Distance Function). En esta línea los trabajos de Picazo, Reig y Hernández (2005), Picazo y Prior (2005), son un buen referente a seguir.

\section{Referencias}

ÁLVAREZ P. A. (Coord.). La medición de la eficiencia y la productividad. Madrid: Editorial Pirámide, 2001.

ALLEN, K. DEA in the ecological context: an overview. In: Westermann G. (Ed.) Data

Envelopment Analysis in the Service Sector, Wiesbaden: Gabler Edition Wissenshaft, 1999.

BANKER, R. et al. An introduction to data envelopment analysis with some of their models and its uses. Research in Governmental and Nonprofit Accounting, v. 5, p.125-163, 1989.
BEVILACQUA M.; BRAGLIA M. Environmental efficiency analysis for ENI oil refineries. Journal of Cleaner Production, v.10, p.58-92, 2002.

BURRITT, R.; SAKA, C. Environmental management accounting applications and eco-efficiency: case studies from Japan. Journal of Cleaner Production, n.14, p.1-14, 2005.

CHARNES A.; COOPER, W.W.; RHODES, E. Measuring the efficiency on decision making units. European Journal of Operational Research, v.2, p.429-444, 1978.

Rev. Adm. UFSM, Santa Maria, v. 1, n. 2, p. 193-208, mai./ago. 2008 
CHUNG; FÄRE; GROSSKOPF. Productivity and undesirable outputs: a direccional distance funtion approach. Journal of environmental management, v.51, p.229-240, 1997.

CÔTE, R.; BOOTH, A.; LOUIS, B. Eco-efficiency and SMEs in Nova Scotia, Canada. Journal of Cleaner Production, n.14, p.542-550, 2005.

COURCELLE, C.; KESTEMONT, M; TYTECA,

D. Assessing the economic and environmental performance of municipal solid waste collection and sorting programmes. Waste Management \& Research, v.16, n.3, p.253-263, 1998.

DYCKHOFF, H.; ALLEN, K. Measuring ecological efficiency with data envelopment analysis (DEA). European Journal of Operational Research, v.132, n.2, p. 312-325, 2001.

ERKKO, S.; MELANEN, M.; MICKWITZ, P. ECOefficiency in the finnish EMAS reports-a buzz word? Journal of Cleaner Production, p.799813, 2005.

ERIKSSON, O. et al. Municipal solid waster management from a systems perspective. Journal of Cleaner Production, v.13, p. 241-252, 2005.

FARELL, M. J. The measurement of productive efficiency. Journal of the Royal Statistics Society, Serie A, v.120, n.3, p.253-281, 1957.

FÄRE, R.; GROSSKOPF, S.; LOVELL, C. A. K. Production frontiers. Cambridge: Cambridge University Press, 1994.

FÄRE, R.; GROSSKOPF, S.; LOVELL, C.A.K.; PASURKA, C. Multilateral productivity comparisons when some outputs ara undesirable: a nonparametric approach. The

Review of Economics and Statistics, v.71, n.1, p.90-98, 1989.

HERNÁNDEZ, F.; PICAZO, A. J.; REIG, E. Análisis no parametrico de eficiencia en presencia de output no deseables. Working Paper. Valencia: Instituto Valenciano de Investigaciones económicas, sep. 1997.

INFORME AEMA. Señales medioambientales de la AEMA 2004. Una actualización de la agencia europea de medio ambiente sobre temas específicos. Agencia Europea de Medio
Ambiente. Disponible en: <http://www.gencat. net $>$. Sitio consultado el 26 sep. 2006.

INFORME DEL CONSELLER DE MEDI AMBIENT I HABITATGE - PROGREMIN Acciones de prevención de residuos municipales promulgados para las localidades de Catalunya y Europa. 2005. Disponible en: < http://www.gencat.net>. Sitio consultado el 26 sep. 2006.

JOLLANDS, N.; LERMIT, J.; PATTERSON, A. Agregate eco-efficiency indices for New Zealand: a principal components analysis. Journal of environmental Management, v.73, p. 293-305, 2004.

KORHONEN, P. J.; LUPTACIK, M. ECo-efficiency analysis of power plants: an extension of data envelopment analysis. European Journal of Operational Research, v.154, p. 437-446, 2004. PORTER, M. E.; VAR DER LINDE, C. Green and competitive. Harvard Business Review, v.73, n.5, p. 120-134, 1995.

ROVIRA, M.; PRIOR, D. Eco-efficiency evaluation of spanish municipalities: a nonparametric frontier approach. EAA Congress, Prague, 2004.

PICAZO, A.J.; REIG, E.; HERNÁNDEZ, F.

Directional distance functions and environmental regulation. Resource and Energy Economics, v.27, p.131-142, 2005.

PICAZO, T.; PRIOR, D. Environmental externalities and efficiency measurement. Working paper. Departament de Economía de I'Empresa, Universidad Autónoma de Barcelona, 2005.

RIALP A. Fundamentos teóricos de la organización de empresas: un enfoque interdisciplinario. Madrid: Pirámide, 2003.

SALAS, V. Economía teórica de la empresa. Información Comercial Española, n.611, p.922, jul.1984.

SEIFORD, L.M.; ZHU, J. Modeling undesirable factors in efficiency evaluation. European Journal of Operational Research, v.142, p. 1620, 2002. 
TYTECA, D. On the measurement of the environmental performance of firms: a literature review and a productive efficiency perspective. Journal of Environmental Management, v.46, p.281-308, 1996.

TYTECA, D. Lineal programming models for the measurement of environmental performance of firms: concepts and empirical results. Journal of Producitivity Analysis, v.8, p.175-189, 1997.
WORLD BUSINESS COUNCIL FOR SUSTAINABLE DEVELOPMENT. ECo-efficiency: creating more value with less impact. UK, 2000. Disponible en: < http://www.wbcsd.org > sitio consultado el 10 jul. 2006.

ZOFÍO, J. L.; PRIETO, A. M. Enviromental efficiency and regulatory standards: the case of CO2 emissions from OECD industries. Resource and Energy Economics, v. 23, p.63-83, 2001. 